\title{
Controversias de la cirugía instrumentada y el tratamiento del dolor lumbar por enfermedad degenerativa. Resultados de la evidencia científica
}

\author{
F.J. Robaina-Padrón
}

Unidad del Dolor Crónico y Neurocirugía Funcional. Hospital Universitario de Gran Canaria "Dr. Negrín". Las Palmas de Gran Canaria.

\section{Resumen}

La investigación y desarrollo de nuevas técnicas instrumentadas de la columna vertebral no escapa a los conflictos de interés. La influencia de las fuerzas financieras en el desarrollo de nuevas tecnologías y su inmediata aplicación en la cirugía de la columna demuestran la relación entre los resultados publicados y el soporte de la industria. Incluso autores que han defendido abiertamente las técnicas de fusión vertebral han demostrado su enorme (millones de dólares) implicación a la hora de influir en las normas de revisión y aprobación de nuevos sistemas de cirugía de columna. Las publicaciones científicas y revisiones que han seguido los principios de la "medicina basada en la evidencia", cuando analizan los resultados de la cirugía de la hernia discal, observan cómo todavía no existe ningún trabajo científico correctamente realizado que compare la cirugía y el tratamiento conservador sin ningún tipo de sesgo científico en la recogida de datos. A la hora de analizar los resultados de la cirugía de la columna, debemos valorar lo que se denomina en los medios económicos de la bolsa americana la "burbuja de la cirugía de columna". La literatura científica no muestra evidencias claras en los estudios costo-beneficio de muchas técnicas de cirugía instrumentada de la columna sobre el tratamiento conservador. No se ha demostrado que la cirugía de fusión vertebral y el reemplazo del disco intervertebral sea mejor que el tratamiento conservador. Como vemos, "existen relaciones entre la industria y el dolor lumbar" y "existe una industria del dolor lumbar". No obstante, "el mercado de la cirugía de la columna" sigue aumentando, ya que los pacientes siguen demandando soluciones para resolver su dolor lumbar. La marea de la evidencia científica parece que va en contra de la fusión vertebral en la enfermedad discal degenerativa, el dolor discogénico y el dolor lumbar inespecífico. Después de dos décadas de avances tecnológicos en este campo, el resultado de las fusiones vertebrales es mediocre. Estu- dios epidemiológicos recientes muestran que la " fusión vertebral" debe aceptarse como "un método no probado o experimental para el tratamiento del dolor lumbar crónico". La literatura quirúrgica sobre la fusión vertebral en los últimos 20 años según revisiones Cochrane establece que : 1- ésta es incompleta, no fiable y descuidada; 2- la instrumentación parece que aumenta ligeramente la tasa de fusión; 3- la instrumentación no mejora los resultados clínicos en general, careciéndose de estudios particulares de subgrupos de pacientes. Se precisan estudios aleatorios que comparen los resultados quirúrgicos con la historia natural, el placebo o el tratamiento conservador. Las Guías Europeas para el manejo del dolor crónico lumbar muestran como existe una "fuerte evidencia" que indica que la cirugía compleja y demandante de la columna vertebral lumbar en la que se emplean diferentes formas de instrumentación, no es más efectiva que una simple, más segura y más barata fusión posterolateral sin instrumentación. Actualmente se observa una tendencia hacia la utilización de "técnicas mínimamente invasivas", abandonando las fusiones transpediculares, indicando claramente que debe aplicarse correctamente el conocimiento basado en la evidencia científica acumulado a lo largo de por lo menos, 20 años. Todo ella nos permite recomendar el "abandono de la senda instrumentista" en gran cantidad de indicaciones actuales, y buscar otros caminos en el campo del tratamiento conservador y rehabilitador correctamente aplicados, utilizando antes de la cirugía descompresiva y de fusión vertebral todos los recursos intervencionistas y mínimamente invasivos que actualmente ofrece el tratamiento moderno del dolor lumbar crónico.

PALABRAS CLAVE: Lumbalgia crónica. Enfermedad degenerativa lumbar. Cirugía instrumentada. Evidencia científica.

Controversies of instrumented surgery and pain relief in degenerative lumbar spine. Results of scientific evidence 


\section{Summary}

Investigation and development of new techniques for intrumented surgery of the spine is not free of conflicts of interest. The influence of financial forces in the development of new technologies an its imediately application to spine surgery, shows the relationship between the published results and the industry support. Even authors who have defend eagerly fusion techniques, it have been demostrated that them are very much involved in the revisión of new articles to be published and in the approval process of new spinal tecnologies. When we analize the published results of spine surgery, we must bear in mind what have been call in the "American Stock and Excahnge" as "the bubble of spine surgery". The scientific literature doesn't show clear evidence in the cost-benefict studies of most instrumented surgical interventions of the spine compare with the conservative treatments. It has not been yet demostrated that fusion surgery and disc replacement are better options than the conservtive treatment. It's necessary to point out that at present "there are relationships between the industry and back pain, and there is also an industry of the back pain". Nonetheless, the "market of the spine surgery" is growing up because patients are demanding solutions for their back problems. The tide of scientific evidence seams to go against the spinal fusions in the degenerative disc disease, discogenic pain and inespecific back pain. After decades of advances in this field, the results of spinal fusions are mediocre. New epidemiological studies show that "spinal fusion must be accepted as a non proved or experimental method for the treatment of back pain". The surgical literature on spinal fusion published in the last 20 years following the Cochrane's method stablish that: 1- this is at least incomplete, not realiable and careless; 2 - the instrumentation seems to sligtly increase the fusion rate ; 3 - the instrumentation doesn't improve the clinical results in general, lacking studies in subgroups of patients. We still are needing randomized studies to compare the surgical results with the natural history of the disease, the placebo effect, or the conservative treatment. The European Guidelines for lumbar chronic pain management show a "strong evidence" indicating that complex and demanding spine surgery where different instrumentation is used, is not more effective than a simple, safer and cheaper posterolateral fusion without instrumentation. Recently, the literature published in this field is sending a message to use "minimally invasive techniques", abandon transpedicular fusions and clearly indicating that we must apply the knowledge accumulated at least along the last 20 years based on the scientific evidence. In conclussion, based in recent information, we must recomend the "abandon of the instrumented pathway" in a great number of present indications for degenerative spine surgery, and look for new strategies in the field of rehabilitation and conservative treatments correctly apply, using before the decompressive and instrumented surgery all the interventional and minimally invasive techniques that are presently offer in the field of modem lumbar chronic pain treatment.

KEY WORDS: Chronic low back pain. Degenerative lumbar disease. Spinal instrumentation. Evidence based medicine.

\section{Introducción}

Actualmente estamos asistiendo a un importante movimiento dentro del campo de la medicina en relación a los conflictos de interés y la investigación médica en general. La investigación y desarrollo de nuevas técnicas instrumentadas de la columna vertebral no escapa a estos conflictos de interés. Un artículo reciente publicado en una revista de difusión mundial como es The Back Letter, hace mención al problema. Los conflictos de interés entre los autores de artículos en revistas o presentaciones en congresos, deberían describir cual es su relación con las empresas fabricantes ${ }^{54}$. Así, el conflicto de interés, no sólo hay que mencionarlo, sino indicar en qué forma las compañías remuneran a los autores; royaltis, acciones, honorarios por consulta u otras formas ${ }^{8,10,11}$. La influencia de las fuerzas financieras en el desarrollo de nuevas tecnologías y su inmediata aplicación en la cirugía de la columna ha sido demostrada por Shah ${ }^{48}$, que resalta la relación entre los resultados publicados y el soporte de la industria. Incluso en autores que han defendido abiertamente las técnicas de fusión vertebral, se ha podido demostrar su enorme (millones de dólares) implicación a la hora de influir en las normas de revisión y aprobación de nuevos sistemas de cirugía de columna ${ }^{12,13}$.

Según los datos que se van conociendo, comienza a crecer en la "comunidad de la columna" una sensación de que los datos publicados basados en investigaciones financiadas por las empresas no son completamente creíbles. Además, la sensación negativa se extiende a los actuales sistemas de investigación, ya que se observa que, posiblemente, no han mirado con diligencia los riesgos y beneficios de los nuevos tratamientos y los nuevos instrumentales antes de que éstos lleguen al mercado ${ }^{54}$.

El Presidente de la Internacional Society for the Study of the Lumbar Spine, pone de relieve que "todavía tenemos que demostrar la utilidad de los tratamientos con argumentos basados en la evidencia". Además, de forma muy explícita, profundiza sobre los aspectos de la Seguridad Social de los diferentes países y el enorme gasto acumulado en sistemas de instrumentación para la columna vertebral, que 
pudieran afectar en el futuro a las inversiones en sanidad ${ }^{49}$. Un estudio recientemente presentado por la Sociedad Española del Dolor (SED) el día del Dolor en Noviembre de 2005, demuestra la importancia que el dolor lumbar tiene en la población española. El 80\% de ella sufrirá dolor lumbar al menos una vez en la vida. Los costes totales asociados a este problema en España superan los 6.000 millones de euros al año.

Nuevas publicaciones nacionales e internacionales cuestionan abiertamente las indicaciones y resultados de la cirugía instrumentada en la patología degenerativa de la columna lumbar. A nivel nacional, Gómez de la Riva $\mathrm{y}$ cols. ${ }^{15}$, describen cuales son las indicaciones actuales para las reintervenciones y fusiones instrumentadas en pacientes operados de columna lumbar por estenosis de canal. Además, los mismos autores, ponen de manifiesto, las controversias existentes en las indicaciones de descompresión y fusión instrumentada en la estenosis de canal y la espondilolistesis degenerativa. A nivel Internacional, recientemente, una revista de enorme prestigio y especializada en dolor como es Pain, (Journal de la IASP; International Association for the Study of Pain), publica dos artículos que claramente ponen en evidencia las controversias existentes entre la cirugía y el tratamiento conservador en la enfermedad degenerativa lumbar pre y postquirúrgica. Paradójicamente, los tratamientos cognitivos y el ejercicio dirigido pueden obtener mejores resultados que la instrumentación vertebral, sobre todo en la columna operada por hernia discal lumbar5,24.

Desde hace años, todos aquéllos que hemos desarrollado nuestra labor asistencial como neurocirujanos pero participando directa o indirectamente en las actividades de una Unidad de Dolor Crónico (UDC), o tratando pacientes con dolor crónico en el seno de los Servicios de Neurocirugía, nos hemos visto enfrentados con el estudio, diagnóstico y tratamiento de síndromes de dolor crónico lumbar secundarios a intervenciones quirúrgicas por hernia discal o estenosis de canal. La búsqueda de soluciones terapéuticas para estos pacientes con síndromes de dolor postlaminectomía o dolor lumbar crónico, constituye actualmente uno de los aspectos fundamentales de la carga de trabajo actual de gran parte de las UDC y de muchos Servicios de Neurocirugía con especial interés en la patología degenerativa de la columna vertebral.

Las revisiones y puesta al día de estos problemas en España, añadiendo algunos aspectos de la experiencia personal, han puesto de manifiesto las malas sensaciones subjetivas que rondan al analizar los resultados publicados y compararlos con los propios ${ }^{15,43-46}$. Cuando consultamos la bibliografía disponible siguiendo criterios de selección y randomización apropiados, parece que se confirman esas malas sensaciones, a la luz de los resultados publicados siguiendo los criterios de la "medicina basada en la evi- dencia ". Cuando se analizan los resultados de la cirugía de la hernia discal, se observa, cómo no existe todavía en la literatura mundial ningún trabajo científico correctamente realizado que compare la cirugía y el tratamiento conservador sin ningún tipo de sesgo científico en la recogida de datos Por esa razón se siguen buscando soluciones no quirúrgicas a este problema $a^{2,3,6,17,20,23,52}$.

\section{Discusión}

A la hora de analizar los resultados de la cirugía de la columna lumbar degenerativa, y siguiendo las indicaciones de autores que han investigado el problema en profundidad, uno de los aspectos fundamentales que debemos tener siempre en cuenta es lo que en los medios económicos de la "Bolsa Americana" se denomina "la burbuja de la cirugía de columna". Debido al lanzamiento de nuevos productos para la instrumentación de la columna, se estima, que "el mercado", puede subir un 22\% anual de acuerdo con algunos analistas. Además, tal y como comentan algunos autores, la literatura científica no muestra evidencias claras en los estudios costo-beneficio de muchas técnicas de cirugía instrumentada de la columna vertebral sobre el tratamiento conservador. No ha sido demostrado que la cirugía de fusión vertebral y el reemplazo del disco intervertebral sea mejor que el tratamiento conservador ${ }^{19}$.

Hay que destacar que "existen relaciones entre la industria y el dolor lumbar" y que "existe una industria del dolor lumbar"21. A pesar de todos los condicionantes ya expuestos con anterioridad, "el mercado de la cirugía de la columna" sigue aumentando, ya que los pacientes demandan continuamente soluciones para resolver su "dolor de espalda"19.

En un artículo recientemente publicado en Norteamérica se indica que: "la marea de la evidencia científica parece que va en contra de la fusión espinal en la enfermedad discal degenerativa, el dolor discogénico y el dolor lumbar inespecifico". Según este artículo, que revisa 24 publicaciones recientes, entre las que existen varios "estudios randomizados", no está claro que los resultados de la cirugía de fusión espinal en la enfermedad degenerativa lumbar con tornillos pediculares, cajas intersomáticas y discos artificiales sea mejor que el tratamiento conservador. Ningún tratamiento actual mejora la historia natural de la enfermedad degenerativa lumbar. Tal y como se expresa en ese artículo de revisión : "a pesar de dos décadas de avances tecnológicos, el resultado de las fusiones espinales es mediocre" $" 53$.

Estudios epidemiológicos publicados recientemente en el British Medical Journal afirman que: "la fusión espinal debe aceptarse como un método no probado o experimental" para el tratamiento del dolor lumbar crónico ${ }^{18}$.

La falta de estudios "randomizados" es lo que ha 
motivado que la American Association of Neurological Surgeons (AANS) y el Congress of Neurological Surgeons (CNS), las dos sociedades neuroquirúrgicas más influyentes de Norteamérica, hayan publicado recientemente sus guías para la realización de los procedimientos de fusión en la "enfermedad degenerativa lumbar". Según el grupo de estudio formado por estas dos sociedades, se incluyen dentro de la enfermedad degenerativa lumbar las siguientes entidades: dolor lumbar intratable en ausencia de estenosis o espondilolistesis; hernia discal y radiculopatía; estenosis y espondilolistesis; estenosis sin espondilolistesis. Se analizan resultados y se emiten recomendaciones en relación a las fusiones intersomáticas, los tornillos pediculares, las técnicas con inyecciones, la utilización de los corsés ortopédicos, la monitorización electrofisiológica en la fusión vertebral y el empleo de los estimuladores eléctricos para acelerar la fusión ósea. La evidencia obtenida por todos los estudios revisados por la AANS y el CNS, sólo permiten formular recomendaciones para todas las variedades de tratamiento existentes, ya que no existe evidencia científica al respecto. La mayoría de los artículos publicados sobre fusión vertebral en la literatura mundial no pudieron incluirse en la revisión, al carecer de criterios válidos de evidencia científica ${ }^{16,25-41}$.

La revista Spine, una de las publicaciones de mayor difusión mundial sobre la patología médica y quirúrgica de la columna vertebral, revista oficial de 14 sociedades nacionales e internacionales, ha publicado hace muy poco tiempo un artículo de revisión sobre la fusión en la enfermedad degenerativa lumbar siguiendo las guías de la Fundación Cochrane ${ }^{14}$. Esta revisión incluye todas las formas de tratamiento quirúrgico de la patología degenerativa que afecta a la columna lumbar. Según esta revisión, se considera patología degenerativa lumbar a las siguientes entidades nosológicas: enfermedad degenerativa discal, inestabilidad, estenosis de canal, y espondilolistesis degenerativa. Este artículo pone de manifiesto que en estos momentos, la estenosis de canal lumbar es la causa principal del aumento de la cirugía vertebral en pacientes mayores de 65 años. Según la misma revisión, dos estudios de meta-análisis revisados son retrospectivos y no aportan datos sobre los criterios diagnósticos, la historia natural, los criterios de indicación quirúrgica y la técnica quirúrgica elegida, entre otras carencias. Los resultados de dos estudios recientes que optan uno por la indicación quirúrgica y otro por el tratamiento conservador, son contradictorios. A pesar de la mayor fusión ósea conseguida con la instrumentación, la mejoría clínica es marginal. No es posible establecer por lo tanto conclusiones acerca de la influencia de la instrumentación y de cada tipo de instrumentación en particular, sobre el resultado quirúrgico obtenido en las diferentes patologías degenerativas de la columna lumbar ${ }^{14}$.

La literatura quirúrgica sobre la fusión espinal en la enfermedad degenerativa en los últimos 20 años según los autores de la revisión Cochrane es: 1- incompleta, no fiable y descuidada; 2- la instrumentación parece que aumenta ligeramente la tasa de fusión; 3- la instrumentación no mejora los resultados clínicos en general, careciéndose de estudios en particular de subgrupos de pacientes ${ }^{4}$.

La evidencia científica acumulada actualmente es, por tanto, insuficiente para analizar los resultados clínicos obtenidos después de la cirugía instrumentada en la enfermedad degenerativa lumbar. Se precisan nuevos estudios científicos que aumenten la evidencia sobre la eficacia y sobre el costo/beneficio de las cirugías descompresivas en la patología lumbar degenerativa, con o sin instrumentación. Se necesitan todavía estudios aleatorios que comparen los resultados quirúrgicos con la historia natural, el placebo o el tratamiento conservador ${ }^{14}$. Existe una "evidencia moderada" que sugiere que los resultados de la cirugía son similares a los resultados obtenidos mediante un programa combinado de ejercicios y terapia cognitiva ${ }^{24}$. Las Guías Europeas para el manejo del dolor crónico lumbar muestran cómo existe una "fuerte evidencia" que indica que la cirugía compleja y demandante de la columna vertebral lumbar en la que se emplean diferentes formas de instrumentación vertebral no es más efectiva que una simple, más segura y más barata fusión posterolateral sin instrumentación ${ }^{51}$.

Otros estudios recientes ponen de manifiesto cómo se está produciendo una tendencia hacia la utilización de "técnicas mínimamente invasivas" en muchos casos de patología degenerativa lumbar, concretamente en la cada vez más frecuente estenosis de canal, cuestionando seriamente la indicación de las descompresiones e instrumentaciones transpediculares. Así, dispositivos interespinosos que en algunos casos pueden implantarse simplemente con anestesia local, alivian los síntomas de la estenosis de canal con claudicación intermitente neurógena sin tener que recurrir a grandes descompresiones y fusiones instrumentadas. Estas técnicas mínimamente invasivas disminuyen de forma muy significativa el riesgo quirúrgico de la cirugía descompresiva con instrumentación y fusión, con su mortalidad y morbilidad asociadas en este grupo cada vez más elevado de pacientes mayores de 65 años y, además, disminuyendo claramente los costos quirúrgicos, al no ser preciso el empleo de instrumentación transpedicular ${ }^{42,55}$.

La claudicación intermitente de origen neurógeno secundaria a estenosis de canal aumentará su prevalencia debido al aumento de las expectativas de vida de la población, teniendo en cuenta que el 13-14\% de todos los pacientes con lumbalgia persistente que acuden a un especialista presentan estenios de canal. Los costos a la sociedad de este tipo de pacientes pueden ser millonarios. El resultado de la cirugía descompresiva en estos caso es muy variable, asociándose al procedimiento quirúrgico tasas elevadas de 
complicaciones y reoperaciones ${ }^{55}$. La génesis del concepto de un simple implante interespinoso para aliviar los síntomas de este grupo de pacientes vino de la observación de los datos clínicos, ya que estos pacientes alivian los síntomas en flexión y empeoran al estar en pie o adoptar una postura en extensión ${ }^{1}$.

Es muy preocupante que en el grupo de pacientes con estenosis de canal lumbar y claudicación intermitente, si los neurocirujanos y ortopedas de columna no aceptan los conceptos de la cirugía mínimamente invasiva para aplicarlos a los pacientes mayores de 65-70 años, éstos pueden seguir siendo intervenidos quirúrgicamente mediante cirugías ineficaces y/o peligrosas para su integridad física. En bastantes ocasiones se detectan también pacientes que han sido rechazados para la cirugía por neurocirujanos y ortopedas de columna, alegando el peligro que representan las técnicas clásicas de descompresión y/o instrumentación vertebral, por la larga duración de las mismas y el riesgo de anemia, infección o pseudoartrosis entre otras complicaciones. Estos pacientes, ancianos, muchos de ellos en excelentes condiciones físicas y mentales, si no son operados al fracasar las técnicas de alivio del dolor, se convierten en adictos a los opiáceos, frecuentadores del sistema sanitario, experimentan un rápido deterioro físico y mental y sobrecargan a sus familiares. No se les ofrecen en muchas ocasiones las nuevas técnicas mínimamente invasivas, mejor toleradas y con muchísima menor mortalidad y morbilidad. Todo ello nos lleva al punto en el que cuando existe un conocimiento médico sedimentado y éste no es aplicado correctamente, se puede caer inevitablemente en el campo de la negligencia médica y del atentado bioético.

Los últimos estudios presentados en la Internacional Society for the Study of the Lumbar Spine destacan que, en estos momentos, se han identificado 14 ensayos clínicos que demuestran que la instrumentación puede mejorar la tasa de fusión vertebral pero no influye en absoluto en el resultado clínico. Las cifras que se manejan en el mercado de la columna ronda los 7 billones de dólares anuales sin que exista evidencia de que realmente se ayuda a los pacientes ${ }^{22}$.

Las técnicas microquirúrgicas realizadas con el microscopio quirúrgico o con los endoscopios modernos como son las foraminotomías y/o minilaminectomías aplicadas a la descompresión de los elementos nerviosos sin fijación pedicular en los casos de estenosis de canal y/o foraminal, comienzan a preconizarse en lugar de las grandes descompresiones e instrumentaciones que han venido realizándose profusamente a lo largo de los últimos 20 años en este tipo de patología ${ }^{50}$.

Existe un enorme interés en el papel que puedan jugar en el futuro inmediato las proteínas morfogenéticas y la terapia génica aplicada a la patología degenerativa de la columna vertebral, dados los escasos resultados obtenidos con las fusiones vertebrales ${ }^{7,9,47}$.

Nachemson, uno de los autores mas importantes en el campo del tratamiento conservador de la enfermedad degenerativa lumbar, comenta que en la cirugía instrumentada de columna degenerativa, "existen alternativas a la medicina basada en la evidencia" que, con frecuencia, lamentablemente, se han seguido por todos nosotros en múltiples ocasiones. Así, tenemos, la " medicina basada en la eminencia", en la que predomina la experiencia del maestro, en la que los mismos errores se cometen una y otra vez. Existe la "medicina basada en la evidencia de los medios de comunicación", orientada hacia los últimos adelantos que son presentados por expertos en las noticias de la mañana. Tenemos también "la medicina basada en la evidencia del mercado", donde confiamos en los anuncios que se publican en las revistas de la especialidad, y, finalmente, tenemos la "medicina basada en la evidencia del beneficio económico", que no precisa aclaración ${ }^{22}$.

\section{Conclusiones}

La importancia de todo lo aquí descrito no estriba solamente en la reducción de la mortalidad, la morbilidad y el gasto económico asociado a la cirugía instrumentada de la columna lumbar degenerativa, que como se ve puede ser muy importante, sino que nos indica claramente que debemos aplicar correctamente el conocimiento basado en la evidencia científica acumulada a lo largo de, por lo menos, 20 años. Todo ello, nos permite, recomendar el "abandono de la senda instrumentadora" en gran cantidad de indicaciones actuales en la enfermedad degenerativa lumbar y buscar otros caminos para aliviar la sintomatología, como son el tratamiento conservador y rehabilitador correctamente aplicados, utilizando antes de la cirugía descompresiva y de fusión vertebral todos los recursos intervencionistas que actualmente ofrece el tratamiento moderno del dolor crónico de origen vertebral. Igualmente, tal y como se establece en los algoritmos modernos de manejo de la lumbalgia crónica, deben aplicarse las nuevas técnicas quirúrgicas mínimamente invasivas antes de pensar en una fusión vertebral instrumentada por vía transpedicular, ya que puede concluirse, que a la luz de los estudios basados en la evidencia científica actual, no se obtienen buenos resultados con las técnicas clásicas de instrumentación transpedicular y fusión en la patología degenerativa de la columna lumbar.

No olvidemos, nuevamente, que los fantasmas de la negligencia médica y los atentados bioéticos planean sobre todos los que nos dedicamos al manejo de este tipo de pacientes. 


\section{Bibliografía}

1. Andersosn, P., Tribus, C., Kitchel, S.: Treatment of neurogenic claudication by interspinous decompression: application of the $\mathrm{x}$-stop device in patients with lumbar degerative spondylolisthesis. J Neurosurg Spine 2006; 4: 464-471.

2. Andreula,, C., Simonetti, L., de Santis, F., et al.: Minimally invasive oxigen-ozone therapy for lumbar disc herniation. American Journal of Neuroradiology 2003; 24: 996-1000.

3. Bonetti, M., Fontana, A., Cotticelli, B., et al.: Intraforaminal $\mathrm{O}_{2}-\mathrm{O}_{3}$ versus periradicular steroidal infiltrations in lower back pain. Randomised controlled study. American Journal of Neuroradiology 2005; 26: 996-1000.

4. Bono, C., Lee, C.K.: Critical análisis of trens in fusion for degenerative disc disease over the past 20 years: Influence of technique of fusion rate and clinical outcome. Spine 2004; 29: 455-463.

5. Brox, J., Reikeras, O., Nygaard, O., et al.: Lumbar instrumented fusion campared with cognitive intervention and exersices in patients with chronic back pain after previous surgery fos disc herniation: A prospective randomized controlled study. Pain 2006; 122: 145-155.

6. Buric, J., Molino Lova, R.: Ozone Chemonucleolysis in nonconrtained lumbar disc herniations: A pilot study with 12 month follow-up. Acta Neurochirurgica 2005; 92: 93-97.

7. Burkus, J., Transfeldt, E., Kitchel, S., et al.: Clinical and radiographical outcomes of anterior lumbar interbody fusion using recombinant human bone morphogenetic prortein-2. Spine 2002; 27: 2396-2408.

8. Carragee, E.: The surgical treatment of disc degeneration: is the race not the swift? Spine Journal 2005; 5: 587588.

9. Cha, C., Boden, S. : Gene therapy applications for spine fusion. Spine 2003; 28: 74-78.

10. Deyo, R.: Spinal-fusion surgery the case for restraint. New England Journal of Medicine 2004; 5: 722-726.

11. Deyo, R.: Leytter to the editor. Spine Journal 2005; 5: 698-699.

12. Errico, T.: A fair balanced view of spine fusion surgery. Spine Journal 2005; 5: 698-699.

13. Errico, T.: Reply to Drs Deyo, Mirza and Nachemson. Spine Journal 2005; 5: 699-700.

14. Gibson, J., Waddell, G.: Surgery for degenerative lumbar spondylosis: Updated Cochrane Review. Spine 2005; 30: 2312-2320.

15. Gómez de la Riva, A., Isla, A., Pérez-López, C., et al.: Causas de reintervención quirúrgica en pacientes con fijación lumbar intervenidos por estenosis de canal. Neurocirugía 2006;17: 232-239.

16. Heary, R.: Introduction to the Guidelines for the performance of fusion procedures for degenerative disease of the lumbar spine. J Neurosurg Spine 2005; 2: 636.

17. Karppinen, J., Coronen, T, Malvivaara, A., et al.:
Tumor necrosis factor-alfa monoclonal antibody, inflimax, used to manage severe ciática. Spine 2003; 28: 750-753.

18. Koes, B.: Surgery versus intensive rehabilitation programs for chronic low back pain. BMJ 2005; 330: 12201221.

19. Lieberman, I.: Disc bulge bubble: Spine economics 101. Spine Journal 2004; 4: 609-613.

20. Muto, M., Andreula, C., Leonardi, M.: Treatment of herniated lumbar disc by intradiscal and intraforaminal oxigen-ozone inyection. Journal of Neuroradiology 2004; 31: 183-189.

21. Nachemson, A.: Industry and back pain and the back pain industry. International Society for the Study of the Lumbar Spine. New York; 2005. The Back Letter 2005; 20: 8 .

22. Nachemson, A.:. Heed the evidence ! The Back Letter 2005; 20: 106-107.

23. Paradiso, R., Alexandre, A.: The different outcomes of patients with disc herniation treated by microdiscectomy or by intradiscal ozone injection. Acta Neurochirurgica 2005; 92: 139-142.

24. Polomano, R., Marcotte, P., Farrar, J.: Spinal fusion and cognitive intervention? In search of the answers. Pain 2006; 122: 4-5.

25. Resnick, D., Choudhri, T., Dailey, A., et al.: Guidelines for the performance of fusion procedures for degenerative disease of the lumbar spine. Part 1: introduction and methodology. J Neurosurg: Spine 2005; 2: 637-638.

26. Resnick, D., Choudhri, T., Dailey, A., et al.: Guidelines for the performance of fusion procedures for degenerative disease of the lumbar spine. Part 2: assessment of functional outcome. J Neurosurg: Spine 2005; 2: 639-646.

27. Resnick, D., Choudhri, T., Dailey, A., et al.: Guidelines for the performance of fusion procedures for degenerative disease of the lumbar spine. Part 3: assessment of economic outcome. J Neurosurg: Spine 2005; 2: 647-652.

28. Resnick, D., Choudhri, T., Dailey, A., et al.: Guidelines for the performance of fusion procedures for degenerative disease of the lumbar spine. Part 4: radiographic assessment of fusion. J Neurosurg: Spine 2005; 2: 653-657.

29. Resnick, D., Choudhri, T., Dailey, A., et al.: Guidelines for the performance of fusion procedures for degenerative disease of the lumbar spine. Part 5: correlation between radiographical and functional outcome. J Neurosurg: Spine 2005; 2: 658-661.

30. Resnick, D., Choudhri, T., Dailey, A., et al.: Guidelines for the performance of fusion procedures for degenerative disease of the lumbar spine. Part 6: magnetic resonance imaging and discography for patient selection for lumbar fusion. $\mathrm{J}$ Neurosurg: Spine 2005; 2: 662-669.

31. Resnick, D., Choudhri, T., Dailey, A., et al.: Guidelines for the performance of fusion procedures for degenerative disease of the lumbar spine. Part 7: intractable low-back 
pain without stenosis or spondylolisthesis. J Neurosurg: Spine 2005; 2: 670-672.

32. Resnick, D., Choudhri, T., Dailey, A., et al.: Guidelines for the performance of fusion procedures for degenerative disease of the lumbar spine. Part 8: lumbar fusion for diusc herniation and radiculopathy. J Neurosurg: Spine 2005; 2: 673-678.

33. Resnick, D., Choudhri, T., Dailey, A., et al.: Guidelines for the performance of fusion procedures for degenerative disease of the lumbar spine. Part 9: fusion in patients with stenosis and spondylolisthesis. J Neurosurg: Spine 2005; 2: 679685.

34. Resnick, D., Choudhri, T., Dailey, A., et al.: Guidelines for the performance of fusion procedures for degenerative disease of the lumbar spine. Part 10: fusion following decompression in patients with stenosis without spondylolisthesis. $\mathrm{J}$ Neurosurg: Spine 2005; 2: 686-691.

35. Resnick, D., Choudhri, T., Dailey, A., et al.: Guidelines for the performance of fusion procedures for degenerative disease of the lumbar spine. Part 11: interbody techniques for lumbar fusion. J Neurosurg: Spine 2005; 2: 692-699.

36. Resnick, D., Choudhri, T., Dailey, A., et al.: Guidelines for the performance of fusion procedures for degenerative disease of the lumbar spine. Part 12: pedicle screw fixation as an adjunct to posterolateral fusion for low-back pain. J Neurosurg: Spine 2005; 2: 700-706.

37. Resnick, D., Choudhri, T., Dailey, A., et al.: Guidelines for the performance of fusion procedures for degenerative disease of the lumbar spine. Part 13: injection therapies, low-back pain, and lumbar fusion. J Neurosurg: Spine 2005; 2: 707-715.

38. Resnick, D., Choudhri, T., Dailey, A., et al.: Guidelines for the performance of fusion procedures for degenerative disease of the lumbar spine. Part 14: brace therapy as an adjunct to or substitute for lumbar fusion. J Neurosurg: Spine 2005; 2: 716-724.

39. Resnick, D., Choudhri, T., Dailey A., et al.: Guidelines for the performance of fusion procedures for degenerative disease of the lumbar spine. Part 15: electrophysiological monitoring and lumbar fusion. J Neurosurg: Spine 2005; 2: 725-732.

40. Resnick, D., Choudhri, T., Dailey A., et al.: Guidelines for the performance of fusion procedures for degenerative disease of the lumbar spine. Part 16: bone graft extenders and substitutes. J Neurosurg: Spine 2005; 2: 733-736.

41. Resnick,D., Choudhri, T., Dailey, A., et al.: Guidelines for the performance of fusion procedures for degenerative disease of the lumbar spine. Part 17: bone grow stimulators and lumbar fusion. J Neurosurg: Spine 2005; 2 : 737-740.

42. Richards, J., Majumdar, S., Derek, P., et al.: The treatment mechanism of an interspinous process implant for lumbar neurogenic intermitted claudication. Spine 2005; 30,
744-749.

43. Robaina, F.: Manejo intervencionista de la ciática y del dolor lumbar postquirúrgico. Revista de la Sociedad Española del Dolor 1999; 6: 233-237.

44. Robaina-Padrón F.: Tratamiento quirúrgico del dolor de espalda. Surgical treatment of back pain. Actualizaciones en Dolor 2000; 1: 42-50.

45. Robaina, F.: Aspectos neuroquirúrgicos del tratamiento del dolor crónico en el tercer milenio. Las Unidades Multidisciplinarias del Dolor. Revista de la Sociedad Española del Dolor 2003; 10: 481-507.

46. Robaina, F.: Situación actual de la cirugía de la columna vertebral degenerativa aplicada al manejo del dolor lumbar crónico. Estenosis de canal. Discopatía degenerativa, resultados basados en la evidencia científica. Rev. Soc. Esp. Dolor 2006; 3: 167-172.

47. Sanden, B., Olerud, C., Petren-Mallmin, M., et al.: Hydroxiapatite coating improves fixation of pedicle screws. A clinical study. J Bone Joint Surg Br 2002; 84: 387-391.

48. Shah, R.: Industry support and correlation to study outcome for papers published in Spine. Spine 2005; 30: 10991104.

49. Szpalski, M.: Spine care in global world. A duality of Priorities. Spine 2006; 31: 1515-1519.

50. Thomé, C., Zevgaridis, D., Leheta., et al.: Outcome after less-invasive decompression of lumbar spinal stenosis: a randomized coparison of unilateral laminotomy, bilateral laminotomy and laminectomy. J Neursurg: Spine 2005; 3: 129-141.

51. Van Tulder, M., Muller, G., Balagué, F., et al.: European guidelines for the management of the chronic nonspecific low back pain. European Commission Research Directorate General Cost Action B 13 Low Back: Guidelines for Its Management 2005.

52. Wiesel, S.: Lost in the Ozone Again. The Back Letter 2005.; 20: 66-67.

53. Wiesel, S.: Massive Spinal treatment industry on increasingly shaky ground? The Back Letter 2005; 20.

54. Wiesel, S.: Conflicts in spinal reserach. The back Letter 2006; 21: 37-41.

55. Zucherman, J., Hsu, K., Hartjen, C., et al.: A Multicenter, prospective, randomised trial evaluating the $\mathrm{X}$ Stop interespinous process decompression system for the treatment of neurogenic intermittent claudication. Two-year follow-up results. Spine 2005; 30: 1351-1358.

Robaina-Padrón, F.J.: Controversias de la cirugía instrumentada y el tratamiento del dolor lumbar por enfermedad degenerativa. Resultados de la evidencia científica. Neurocirugía 2007; 18: 406-413.

Correspondencia postal: Dr. Francisco Javier Robaina Padrón. Jefe de la Unidad del Dolor Crónico y Neurocirugía Funcional. 
Nivel 5. Hospital Universitario de Gran Canaria "Dr. Negrín".

Comentario al trabajo Controversias de la cirugía instrumentada y el tratamiento del dolor lumbar por enfermedad degenerativa. Resultados de la evidencia científica de F. Robaina.

El Dr. Robaina muestra en su artículo la escasa relación de la medicina basada en la evidencia con los resultados aceptables obtenidos con las cirugías instrumentadas, proponiendo otras alternativas de tratamiento mas conservador o mínimamente invasivos que podían ser utilizados en gran medida .

Estoy de acuerdo con el autor que conseguir estudios randomizados, aleatorios y a doble ciego sobre los resultados de las instrumentaciones es bastante complicado. También es cierto que el interés económico y el mundo de empresas que soportan este material de instrumentaciones para artodesis es importante como el autor comenta, pero no es menos cierto que las empresas que dan soporte al tratamiento médico conservador son también importantes, ya que supone un gran gasto farmacéutico en el tratamiento del dolor crónico espinal de estos pacientes.

Por otra parte, teniendo en cuenta que es una enfermedad degenerativa que puede ocurrir en personas jóvenes, pero aún más en personas mayores y que cada día más se
Barranco de la Ballena s/n. 35010 Las Palmas de Gran Canaria. exige por la sociedad en la que vivimos mayor calidad de vida, es necesario tener una amplia batería de tratamientos buscando alternativas desde las más conservadoras con analgésicos y rehabilitación, pasando por técnicas percutáneas y mínimamente invasivas (como apunta el autor), sin olvidarse de las técnicas quirúrgicas que pueden parecer mas cruentas como son las artrodesis con instrumentaciones. Para ello debemos de intentar hacer un buen diagnóstico para aplicar lo mejor posible las diferentes pautas de tratamiento. También hay que hacer constar de qué disponemos para el tratamiento nosotros mismos y plantearnos el buscar desde el principio qué va a ser lo mejor para el paciente, ya que en un amplio porcentaje precisarían de un grupo multidisciplinar para conseguir un resultado mas completo y efectivo en la patología espinal de origen degenerativo. 